\title{
Nonlocal and nonlinear effects in shock waves
}

\author{
David Jou* and Diego Pavón \\ Department of Physics, Faculty of Sciences, Autonomous University of Barcelona, 08193 Bellaterra, Barcelona, Spain
}

(Received 29 April 1991)

\begin{abstract}
The formulation of generalized transport laws leading to causal hyperbolic hydrodynamical equations gives rise to a critical Mach number $M_{c}$ at which a singularity develops in the shock wave layer. This happens in kinetic theory of gases (Grad's 13 moments method) and extended irreversible thermodynamics as well. In this paper, it is shown that if nonlinear and nonlocal effects are taken into account, $M_{c}$ significantly increases. Specifically, nonlocal effects raise $M_{c}$ from 2.01 up to 3.03. Further consideration of some nonlinear terms raise $\boldsymbol{M}_{c}$ from the latter value up to 4.67.
\end{abstract}

PACS number(s): 47.40.Nm, 05.70.Ln

\section{INTRODUCTION}

The analysis of high-frequency and short-wavelength phenomena has fostered the progress of nonequilibrium statistical mechanics and nonequilibrium thermodynamics. In the mentioned conditions, the classical transport laws, namely, Fourier's law of heat conduction and Newton-Stokes law of viscous pressure, must be substituted by more general expressions taking into account nonlocal effects, both in time and space. Furthermore, if the amplitude of the perturbation is high enough, nonlinear dissipative effects must be also considered. The derivation and interpretation of such general equations is one of the main topics of research in nonequilibrium phenomena.

At high frequencies, one cannot take as independent hydrodynamical variables only the conserved, or slow, variables, as mass, momentum, and energy, but one must include in the set of basic variables those whose relaxation time is of the order of the inverse of the frequency. Thus, some nonconserved variables, as, for instance, dissipative flows (heat flow, viscous pressure tensor, and so on) may become independent variables at high frequencies. This idea is the basis of some microscopic developments, as the 13-moments method in kinetic theory of gases [1], some formulations of generalized hydrodynamics based on projection-operator techniques [2], and the macroscopic theory known as extended (or transient) irreversible thermodynamics (EIT), [3-10]. All these developments include as independent variables the usual dissipative flows.

The evolution equations for the flows play in these theories the role of generalized transport equations. Due to the enormous complexity of real systems, the simple evolution equations proposed in these theories are no more than plausible caricatures which allow us to appreciate qualitative effects beyond the classical theory as, for instance, the high-frequency behavior of thermal waves $[3,11]$ or of shear waves, the nonequilibrium corrections to hydrodynamic noise at high frequencies [12], nonequilibrium modifications of the equations of state and their consequences on the critical point [13], nonequilibrium contributions to the entropy [3-10], etc. Such modifications are of interest in the relativistic context too, where the infinite speed of thermal and viscous signals predicted by the classical transport equations are no longer admissible [14].

Shock waves have been used as a testing ground of such generalized theories as nonlocal effects become very important there. Both Grad's kinetic model and EIT predict the breakdown of the regular structure of the shock layer beyond a critical Mach number [15] (1.65 or 2.07 , depending on the model) which is at variance with experimental and numerical results for these show a regular structure up to Mach numbers as high as 4 [16] and 10 [17], respectively. This has prompted the comment that the EIT is currently a theory in crisis [18].

Heuristically, this feature is quite general in hyperbolic systems where smooth signals cannot propagate at speeds higher than the highest characteristic speed [1]. In contrast, the usual parabolic (classical) theory does not yield any critical Mach number but it allows us, in principle, to evaluate the shock thickness up to arbitrarily high Mach numbers. Accordingly, it seems wise to examine the existing formulations of EIT in order to improve, as far as possible, its connection with experiments, i.e., to increase the critical Mach number predicted by them.

The purpose of this paper is to reexamine this interesting problem from the point of view of the more recent formulation of EIT. Indeed, the simple older models of EIT include as additional independent variables the classical dissipative flows only. However, at frequencies comparable to the inverse of the collision time an infinite number of variables should be included in the theory as, for instance, higher and higher-order moments of the distribution function. In fact, the relaxation times of these nonconserved variables cannot be shorter than the collision time, so that they should be considered as independent variables in these conditions (a development of EIT with the inclusion of higher-order flows has been already undertaken [19]). Since the departure with respect to equilibrium is important in shock waves nonlinear effects are expected to be very relevant. Here we will show that their inclusion substantially increases the critical Mach number.

The plan of the paper is as follows. The next section is 
devoted to a brief review of the analysis of shock waves in the framework of the older model of EIT. In Secs. III and IV spatial nonlocalities and dissipative nonlinearities are considered, respectively. Finally, in Sec. V, the main conclusions are presented.

\section{SHOCK WAVES IN THE OLD VERSION OF EIT}

In this section we briefly synthesize the main ideas of the old version of EIT. The subject has been extensively reviewed $[3,8]$ where the reader interested in details is referred to the bibliography. Here it is sufficient to state that EIT, in its barest version, rests on the following hypotheses: (1) It exists as a generalized entropy function which depends not only on the classical variables but on the dissipative flows as well. The latter are considered independent variables on the same foot as the classical ones. (2) Such an entropy function is a maximum at equilibrium state. Its flow may depend on all dissipative flows and its production rate is positive definite.

For unicomponent simple fluids the dissipative flows are taken to be the heat flow $q$ and the traceless viscous pressure tensor $\mathrm{P}^{v}$ (the bulk viscous pressure vanishes identically in ideal monoatomic nonrelativistic gases). The generalized Gibbs equation, the generalized entropy flow and the evolution equation for the flows are

$$
\begin{aligned}
& d s=\theta^{-1} d u+\theta^{-1} \Pi d \vartheta-\frac{\tau_{1} \vartheta}{\kappa T^{2}} \mathbf{q} d \mathbf{q}-\frac{\tau_{2} \vartheta}{2 \eta T} \mathrm{P}^{v}: d \mathrm{P}^{v}, \\
& \mathbf{J}^{s}=\theta^{-1} \mathbf{q}+\beta \mathrm{P}^{v} \cdot \mathbf{q}, \\
& \rho \dot{\mathbf{q}}=-\left(\rho / \tau_{1}\right)(\mathbf{q}+\kappa \nabla T)+\frac{\rho \beta \kappa T^{2}}{\tau_{1}} \nabla \cdot \mathrm{P}^{v} \\
& \rho \dot{\mathrm{P}} v=-\left(\rho / \tau_{2}\right)\left(\mathrm{P}^{v}+2 \eta \stackrel{\circ}{ }\right)+\frac{2 \rho \beta \eta T}{\tau_{2}}(\nabla \cdot \mathbf{q})^{s}
\end{aligned}
$$

with $\kappa$ and $\eta$ the thermal conductivity and shear viscosity, $\tau_{1}$ and $\tau_{2}$ the relaxation times of $\mathbf{q}$ and $\mathbf{P}^{v}$, respectively. $\theta$ and $I I$ denote the generalized absolute temperature and thermodynamic pressure, which reduce to $T$ and $p$, the usual local-equilibrium absolute temperature and thermodynamic pressure for small values of $q$ and $P^{v}$. $\vartheta$ stands for the specific volume $\rho^{-1}$ and $V^{\circ}$ is the traceless symmetric part of the velocity gradient. When $\tau_{1}=\tau_{2}=\beta=0$, (2.1) reduces to the usual Gibbs equation of the local-equilibrium theory, (2.2) becomes the standard entropy flow, and (2.3) and (2.4) reduce to the classical Fourier and Newton-Stokes laws, respectively.

The evolution equations (2.3) and (2.4), together with the classical balance laws of mass, momentum, and energy,

$$
\begin{aligned}
& \dot{\rho}=-\rho \boldsymbol{\nabla} \cdot \mathbf{v}, \\
& \rho \dot{\mathbf{v}}=-\nabla p-\nabla \cdot \mathbf{P}^{v}+\rho \mathbf{F}, \\
& \rho \dot{u}=-\nabla \cdot \mathbf{q}-p \nabla \cdot \mathbf{v}-\mathrm{P}^{v}: \stackrel{\vee}{ },
\end{aligned}
$$

provide us with a complete set of equations for the evolu- tion of the flows.

When Eqs. (2.3) and (2.4) together with (2.5) - (2.7) are used to study thermal and shear waves one obtains for the high-frequency limit of the propagation speed of the corresponding disturbances

$$
c_{1}=\left(\kappa / \rho c \tau_{1}\right)^{1 / 2}
$$

and

$$
c_{2}=\left(\eta / \rho \tau_{2}\right)^{1 / 2}
$$

Note that $\kappa$ and $\eta$ are assumed to be known, so that Eqs. (2.8) can be used, in principle, to obtain the effective values of $\tau_{1}$ and $\tau_{2}$ from the experimental determination of $c_{1}$ and $c_{2}$. Since actual measurements are difficult, one often identifies $\tau_{1}$ and $\tau_{2}$ from kinetic theory (13-moment method, relaxation time approximations) or from second-order contributions to the dispersion relations of ultrasonic waves.

For one-dimensional shock wave, Eqs. (2.3)-(2.7) reduce to

$$
\begin{aligned}
& \frac{d}{d x}(\rho v)=0, \\
& \rho v \frac{d v}{d x}+\frac{d P_{x x}^{v}}{d x}+\frac{d p}{d x}=0, \\
& \rho v \frac{d u}{d x}+\frac{d q}{d x}+p \frac{d v}{d x}+P_{x x}^{v} \frac{d v}{d x}=0, \\
& q+\kappa \frac{d T}{d x}+\tau_{1} v \frac{d q}{d x}-\beta \kappa T^{2} \frac{d P_{x x}^{v}}{d x}=0, \\
& P_{x x}^{v}+\frac{4}{3} \eta \frac{d v}{d x}+\tau_{2} v \frac{d P_{x x}^{v}}{d x}-\frac{4}{3} \beta \eta T \frac{d q}{d x}=0,
\end{aligned}
$$

where $q=q_{x}, P^{v} \equiv P_{x x}^{v}$, and $v=v_{x}$. These relationships must be supplemented by the state equations

$$
\begin{aligned}
& u=c_{v} T=c^{2}[\gamma(\gamma-1)]^{-1}, \\
& p=n k_{B} T=\rho c^{2} \gamma^{-1},
\end{aligned}
$$

in which we have written $u$ (the internal energy) and $p$ in terms of the Laplace velocity of sound $c$ and the adiabatic coefficient $\gamma$ inasmuch as these expressions are the most suitable ones for the analysis of shock waves. The first three equations can be immediately integrated to give

$$
\begin{aligned}
& \rho v=M^{*}, \\
& \rho v^{2}+P_{x x}^{v}+p=P^{*}, \\
& \rho v\left[u+\frac{v^{2}}{2}\right]+\left(P_{x x}^{v}+p\right) v+q=Q^{*},
\end{aligned}
$$

where $M^{*}, P^{*}$, and $Q^{*}$ are integration constants.

The dissipative flows play an important role in the determination of the spatial structure of the shock layer, i.e., the spatial dependence of the thermodynamic quantities and the velocity across the layer. We next sketch the analysis of Anile and Majorana [15].

Upon introducing the dimensionless variables

$$
\omega \equiv\left(M^{*} / P^{*}\right) v, \quad \phi \equiv\left(p / P^{*}\right),
$$


the combination of Eqs. (2.9)-(2.13) leads to the following equations for the spatial dependence of $\omega$ and $\phi$ :

$$
\begin{aligned}
& a_{11} \frac{d \omega}{d x}+a_{12} \frac{d \phi}{d x}=A_{1} D_{1}, \\
& a_{21} \frac{d \omega}{d x}+a_{22} \frac{d \phi}{d x}=A_{2} D_{2},
\end{aligned}
$$

where

$$
\begin{aligned}
& a_{11}=\left(\frac{5}{2}\right) \phi^{2}+r_{1} \omega^{2}-\left[r_{1}(\gamma-1)^{-1}+r_{2}\right] \omega \phi-r_{1} \omega, \\
& a_{12}=-r_{1}(\gamma-1)^{-1} \omega^{2}+\left(\frac{5}{2}-r_{2}\right) \omega \phi, \\
& a_{21}=\frac{8}{9}\left[1-\frac{2}{5} r_{2}(\gamma-1)^{-1}\right] \phi+\left(\frac{16}{45} r_{2}-r_{3}\right) \omega-\frac{16}{45} r_{2}, \\
& a_{22}=-r_{3} \omega-\frac{16}{45} r_{2}(\gamma-1)^{-1} \omega, \\
& A_{1}=\frac{5}{2}\left(M^{*} / P^{*}\right)(R p / \kappa m), \quad A_{2}=\frac{2}{3}\left(M^{*} / P^{*}\right)(p / \eta), \\
& r_{1}=\frac{5 \tau_{1}}{2 \rho T \kappa} p^{2}, \quad r_{2}=-\frac{5}{2} p \beta T, r_{3}=\frac{2 p \tau_{2}}{3 \eta}, \\
& D_{1}=(\gamma-1)^{-1} \omega \phi-a^{*}-\frac{1}{2} \omega^{2}+\omega, D_{2}=\omega+\phi-1,
\end{aligned}
$$

$a^{*}=M^{*} Q^{*} / P^{* 2}$

Here, $R$ is the ideal gas constant, and $m$ the mass of the molecules. Note that $a^{*}$ may be written in terms of the properties of the equilibrium state upstream to the shock layer as

$a^{*}=\gamma^{2} M_{-}^{2}\left(1+\gamma M_{-}^{2}\right)^{-2}\left[(\gamma-1)^{-1}+\frac{1}{2} M_{-}^{2}\right]$.

When the determinant $D(\omega, \phi)=a_{11} a_{22} \cdot a_{12} a_{21}$ of the homogeneous part of (2.16) is different from zero the system of Eqs. (2.16) can be written as

$$
\frac{d \omega}{d x}=\frac{\Delta_{1}}{D}, \frac{d \phi}{d x}=\frac{\Delta_{2}}{D},
$$

with $\Delta_{1}=A_{1} D_{1} a_{22}-A_{2} D_{2} a_{12}$ and $\Delta_{2}=A_{2} D_{2} a_{11}$ $-A_{1} D_{1} a_{21}$.

The equilibrium states located far away from the shock layer are given by the condition $\Delta_{1}=\Delta_{2}=0$ or equivalently by $D_{1}=D_{2}=0$, i.e., they are the solution of the system

$$
\begin{aligned}
& (\gamma-1)^{-1} \phi \omega-a^{*}-\frac{1}{2} \omega^{2}+\omega=0, \\
& \omega+\phi-1=0 .
\end{aligned}
$$

This system has two real positive solutions $\boldsymbol{P}_{-}$ $=\left(\omega_{-}, \phi_{-}\right), \quad P_{+}=\left(\omega_{+}, \phi_{+}\right)$whenever $\frac{1}{2}<a^{*}<\frac{1}{2} \gamma^{2}\left(\gamma^{2}\right.$ $-1)^{-1}$. These solutions characterize the equilibrium states at both sides of the shock layer and are given by

$$
\omega_{-}=\frac{\gamma+\epsilon}{\gamma+1}, \phi_{-}=\frac{1-\epsilon}{\gamma+1}
$$

$$
\omega_{+}=\frac{\gamma-\epsilon}{\gamma+1}, \quad \phi_{+}=\frac{1+\epsilon}{\gamma+1},
$$

with

$$
\epsilon=-\gamma\left(1-M_{-}^{2}\right)\left(1+\gamma M_{-}^{2}\right)^{-1},
$$

where $M_{-}=v_{-} / c_{-}$denotes the upstream Mach number and the quantity $\epsilon$ parametrizes the strength of the shock wave. For weak shocks $\left(M_{-} \rightarrow 1\right), \epsilon \rightarrow 0$ and for strong shocks $\left(M_{-} \rightarrow \infty\right), \epsilon \rightarrow 1$.

Up to first order in $\epsilon$, the structure of the shock layer is determined by [15]

$$
\omega_{0}(y)=-\phi_{0}(y)=-(\gamma+1)^{-1} \tanh \left(B^{*} y\right),
$$

where $y=\epsilon x$ and

$$
B^{*-1}=\frac{2}{M^{*}}(\gamma+1)^{-1}[(\gamma-1)(\bar{\kappa} / c)+(4 / 3) \gamma \bar{\eta}]
$$

with $\bar{\kappa}$ and $\bar{\eta}$ the transport coefficients evaluated for $\phi=(\gamma+1)^{-1}$. Note that the relaxation coefficients $r_{1}, r_{2}$, and $r_{3}$ do not appear in (2.23) so that for weak shock waves the width and structure of the shock layer is not influenced explicitly by the relaxation terms. Such an influence appears, however, in higher-order approximations where numerical methods are needed to study the shock profile. In Table I we show the numerical results obtained by Anile and Majorana for the width of the shock layer defined as

$$
L \equiv \frac{\omega_{-}-\omega_{+}}{(d \omega / d x)_{\max }},
$$

in terms of the mean upstream mean free path $l_{-}$. This is usually taken as

$$
l_{-}=\frac{16 \eta_{-}}{5 \rho_{-}}\left(\frac{m}{2 k_{B} T_{-}}\right)^{1 / 2}
$$

The most conspicuous consequence of the relaxation terms in the present version of EIT is to put some limits on the Mach numbers for which the shock layer shows a regular structure. The existence of critical Mach numbers in the extended description is linked to the mathematical property that a regular unique solution to (2.19) can only exist if the determinant $D(\omega, \phi)$ differs from zero. Otherwise $d \omega / d x$ and $d \phi / d x$, as given by Eq. (2.19), will diverge since generally at the points where $D(\omega, \phi)$ vanishes, $\Delta_{1}$ and $\Delta_{2}$ do not. A necessary condition to avoid the occurrence of $D(\omega, \phi)=0$ is that $D\left(\omega_{2}, \phi_{2}\right)$ and $D\left(\omega_{1}, \phi_{1}\right)$ bear the same sign. If they do not there will be an intermediate couple $\omega^{\prime}, \phi^{\prime}$ with $\omega_{2}<\omega^{\prime}<\omega_{1}, \phi_{2}<\phi^{\prime}<\phi_{1}$ such that $D\left(\omega^{\prime}, \phi^{\prime}\right)=0$.

For ideal monoatomic gases $\left(\gamma=\frac{5}{3}\right)$ the determinant $D(\omega, \phi)$ is, according to definitions $(2.17 \mathrm{~b})$,

$$
\begin{aligned}
D(\omega, \phi)=-\omega & {\left[\left[\frac{5}{2} \phi^{2}+r_{1} \omega^{2}-\frac{1}{2}\left(3 r_{1}+2 r_{2}\right) \omega \phi-r_{1} \omega\right]\left(r_{3}+\frac{8}{15} r_{2}\right)\right.} \\
& \left.+\left(\left(5-2 r_{2}\right) \frac{\phi}{2}-\frac{3}{2} r_{1} \omega\right]\left[\frac{8}{45}\left(5-3 r_{2}\right) \phi+\frac{1}{45}\left(16 r_{2}-45 r_{3}\right) \omega-\frac{16}{45} r_{2}\right]\right] .
\end{aligned}
$$


TABLE I. The ratio between the mean free path $l_{-}$and the shock width $L$ is shown for different Mach numbers for three models.

\begin{tabular}{cccc}
\hline \hline $\boldsymbol{M}_{-}$ & Classical model & G I model & G II model \\
\hline 1.1 & 0.0346 & 0.0346 & 0.0347 \\
1.3 & 0.0983 & 0.0981 & 0.0985 \\
1.5 & 0.1527 & 0.1528 & 0.1534 \\
1.7 & 0.1972 & & 0.1989 \\
1.9 & 0.2326 & & 0.2357 \\
\hline \hline
\end{tabular}

We shall consider three models. (i) The classical one, with the Newton-Stokes and Fourier transport laws. For this model one has $r_{1}=r_{2}=r_{3}=0$. (ii) The Grad's 13moments model of kinetic theory $[1,15]$ (G I model) where $r_{1}=r_{2}=1, r_{3}=\frac{2}{3}$. (iii) Model G II with the values $r_{1}=r_{3}=2(5 \pi)^{-1}$ and $r_{2}=0$, obtained by Carrassi and Morro [20] by contrasting the dispersion relation that follows from the set of equations (2.9) - (2.13) with the experimental data on the phase speed of ultrasounds.

Bearing in mind that according to $(2.20 \mathrm{~b}), \omega=1-\phi$, Eq. (2.25) reduces to the following.

Classical model,

$$
D(1-\phi, \phi)=-\frac{20}{9}(1-\phi) \phi^{2} \text {. }
$$

G I model,

$$
D(1-\phi, \phi)=-(1-\phi)\left(9.2 \phi^{2}-7.2 \phi+1\right) .
$$

G II model,

$$
D(1-\phi, \phi)=-(1-\phi)\left(3.09 \phi^{2}-0.58 \phi-0.02\right) \text {. }
$$

Note that the physically significant domain of variation of $\phi$ is, according to $(2.21), \frac{3}{8}(1-\epsilon)<\phi<\frac{3}{8}(1+\epsilon)$, with $0 \leq \epsilon \leq 1$, which implies $0 \leq \phi \leq \frac{3}{4}$. For the classical model $D(\phi)$ is always negative in the mentioned domain. In the G I model $D\left(\omega_{1}, \phi_{1}\right)$ and $D\left(\omega_{2}, \phi_{2}\right)$ are both positive up to $\phi=0.18$, which according to $(2.21)$ corresponds a (critical) Mach number $M_{c}=1.65$. For $1.65<M<1.86$, $D\left(\omega_{2}, \phi_{2}\right)$ is negative whereas $D\left(\omega_{1}, \phi_{1}\right)$ remains positive. In the G II model both $D\left(\omega_{1}, \phi_{1}\right)$ and $D\left(\omega_{2}, \phi_{2}\right)$ are negative for $M$ lower than $M_{c}=2.07$ in which case $D\left(\omega_{2}, \phi_{2}\right)$ remains positive up to $M>2.96$. Thus in the generalized models G I and G II a regular shock layer ceases to exist for $M_{c}(\mathrm{I}) \geq 1.65$ and $M_{c}$ (II $) \geq 2.07$. However, it has been found in experiments with argon that the structure of the shock layer is regular at least up to $M_{-}=4$ and there are indications provided by computer simulations that the regularity may go beyond $M_{-}=10[16,17]$ (for higher $M_{-}$values experiments are very difficult to perform). Hence if a critical Mach number actually exists it should be greater than 4 .

Table I shows that in the common domain of validity the numerical results for the width of the shock layer are very similar for the classical model, the G I model, and the G II model. The trouble is that the models G I and G II, which should be expected to be more accurate than the classical one, fail to describe shock waves for $M>2.1$ where the latter is still successful.
In the next section we show that a possible way out for this problem could be to "renormalize" the transport coefficients in order to take into account higher-order nonlocal and nonlinear effects, ignored in the old version of EIT sketched above.

\section{NONLOCAL EFFECTS}

Relationships (2.3) and (2.4) do not have the general form of a balance equation. For a generic quantity, say $z$, such a form reads

$$
\rho \dot{z}=-\nabla \cdot \mathbf{J}^{z}+\sigma^{z},
$$

with $\mathbf{J}^{z}$ the flow of $z$ and $\sigma^{z}$ the production rate of $z$ per unit volume. The flow of a quantity describes how it spreads in space as response to inhomogeneities, i.e., it describes nonlocal effects.

When $q$ and $P^{v}$ are included in the theory, their respective flows and the flows of these flows should also be included as independent variables, at least in principle. If the relaxation times of the higher-order flows are much shorter than the inverse of the frequency, these variables should not be considered as independent variables, but they should be considered as so otherwise. In monoatomic ideal gases, the relaxation times of the higher-order flows - which correspond to higher-order moments of the distribution function from a microscopic point of view [21] - are all of the same order. Consequently, when $\mathbf{q}$ and $\mathbf{P}^{v}$ have to be considered as independent variables, all their higher-order flows should be considered as independent variables as well. This point of view has been explored in EIT theories [19].

For the sake of simplicity, we shall restrict ourselves to $\mathbf{q}$ (the analysis for $\mathrm{P}^{v}$ is analogous). We denote $\mathbf{q}$ as $\mathbf{q}^{(1)}$, $\mathbf{q}^{(2)}$ is a second-order tensor describing the flow of $\mathbf{q}^{(1)}$ and, in general, $\mathrm{q}^{(n)}$ is the $n$th order tensor corresponding to the flow of $\mathrm{q}^{(n-1)}$, an $(n-1)$ th order tensor.

The evolution equation for $q^{(n)}$ may be written as

$$
\rho \dot{\mathbf{q}}^{(n)}=-\nabla \cdot \mathrm{q}^{(n+1)}+\sigma^{(n)} \text {. }
$$

In EIT the explicit form of the evolution equations compatible with the second law is obtained as follows. One assumes a generalized entropy and a generalized entropy flow of the form [19]

$$
d s=\theta^{-1} d u+\theta^{-1} \Pi d \vartheta-\sum_{n=1}^{\infty} \alpha_{n} \vartheta \mathrm{q}^{(n)} \cdot d \mathrm{q}^{(n)}
$$

and

$$
\mathbf{J}^{s}=\theta^{-1} \mathbf{q}^{(1)}+\sum_{n=1}^{\infty} \beta_{n} q^{(n+1)} \cdot \mathrm{q}^{(n)} .
$$

The entropy production can be derived from the equation of evolution for $s$,

$$
\rho \dot{s}=-\nabla \cdot \mathbf{J}^{s}+\sigma^{s} .
$$

Upon introducing (3.3) and (3.4) into (3.5) and taking into account the balance equations (2.5) and (2.7) it follows

$$
\begin{aligned}
\sigma^{s}= & -\mathbf{q}^{(1)} \cdot\left(\nabla T^{1}+\alpha_{1} \dot{\mathbf{q}}^{(1)}\right) \\
& -\sum_{n=1}^{\infty} q^{(n)} \cdot\left(\alpha_{n-1} \dot{\mathrm{q}}^{(n)}-\beta_{n+1} \nabla \cdot \mathrm{q}^{(n+1)}-\beta_{n} \nabla \mathrm{q}^{(n-1)}\right) .
\end{aligned}
$$


The simplest form of the evolution equation for the flows satisfying (3.1) is

$$
\begin{aligned}
\rho \dot{q}^{(1)}= & -\frac{\rho}{\tau_{1}}\left(\mathbf{q}^{(1)}+\kappa \nabla T\right)+\frac{\rho \beta_{1}}{\alpha_{1}} \nabla \cdot q^{(2)}, \\
\rho \dot{\mathrm{q}}^{(n)}= & -\frac{\rho}{\tau_{n}}\left[q^{(n)}+\frac{\beta_{n-1} \tau_{n}}{\alpha_{n}} \nabla \cdot q^{(n-1)}\right] \\
& +\frac{\rho \beta_{n}}{\alpha_{n}} \nabla \cdot q^{(n+1)} .
\end{aligned}
$$

Since $\mathrm{q}^{(n+1)}$ is the flow of $\mathrm{q}^{(n)}$ one has $\alpha_{n}=-\rho \beta_{n}$.

When written in the Fourier space this set of equations allows us to define a thermal conductivity which depends on the frequency $\omega$ as well as on the wave vector $\mathbf{k}$ as

$$
\kappa(\omega, k)=\frac{\kappa_{0}}{1+i \omega \tau_{1}+\frac{l_{1}^{2} k^{2}}{1+i \omega \tau_{2}+\frac{l_{2}^{2} k^{2}}{1+i \omega \tau_{3}+\frac{l_{3}^{2} k^{2}}{1+\cdots}}},},
$$

with

$$
l_{n}^{2}=\beta_{n} \frac{\tau_{n} \tau_{n+1}}{\alpha_{n} \alpha_{n+1}} .
$$

An asymptotic expression for Eq. (3.9) can be obtained with the use of the scheme proposed by Gianozzi et al. [22]. It yields

$$
\frac{\kappa_{\infty}(\omega, k)}{\kappa_{0}}=\frac{1}{1+i \omega \tau_{\infty}+l_{\infty}^{2} \frac{\kappa_{\infty}(\omega, k)}{\kappa_{0}}},
$$

with $\tau_{\infty}$ and $l_{\infty}$ the values of $\tau_{n}$ and $l_{n}$ in the high- $n$ limit. From the last equation we have

$$
\frac{\kappa_{\infty}(\omega, k)}{\kappa_{0}}=\frac{-\left(1+i \omega \tau_{\infty}\right) \pm\left[\left(1+i \omega \tau_{\infty}\right)^{2}+4 l_{\infty}^{2} k^{2}\right]^{1 / 2}}{2 l_{\infty}^{2} k^{2}}
$$

The dispersion relation for thermal waves reads

$$
\rho c i \omega=-k^{2} \kappa_{\infty}(\omega, k) .
$$

In the high-frequency limit $\left(\omega \gg>\tau^{-1}\right),(3.11)$ yields

$$
i \omega=\chi\left(2 l_{\infty}^{2}\right)^{-1}\left[i \omega \tau_{\infty} \pm\left(4 l_{\infty}^{2} k^{2}-\omega^{2} \tau_{\infty}^{2}\right)^{1 / 2}\right],
$$

with $\chi \equiv \kappa / \rho c$. Hence it follows for the speed of thermal waves

$$
c_{1}^{2}=\frac{\chi^{2}}{l_{\infty}^{2}}\left[\left[\frac{\chi \tau_{\infty}}{l_{\infty}^{2}}\right]-1\right]^{-1}
$$

Comparison of this equation with (2.8a) allows us to define an effective relaxation time as

$$
\tau_{1 \mathrm{eff}}=\tau_{1}-l_{\infty}^{2} \chi^{-1}
$$

We shall take $l_{\infty}=l_{-} / 2$, and $\tau_{\infty}=\tau$, in accordance with Hess [21]. Thus, $l_{\infty}=3 k_{B} T^{2} \tau / 4 m$ where $\tau$ denotes the collision time. If one takes into account that in kinetic theory $\chi=-5 k_{B} T \tau_{1} / 3 m$ one has $\tau_{1 \text { eff }}=4 \tau_{1} / 5$.

A similar development for the speed of shear waves leads to

$$
\tau_{2 \mathrm{eff}}=\tau_{2}-(\rho / \eta) l_{\infty}^{2}
$$

If one considers $l_{\infty}^{2}=3 k_{B} T \tau^{2} / 4 m$ and $\eta=p \tau$ one has $\tau_{2 \text { eff }}=\tau_{2} / 4$.

By replacing $\tau_{1} / \kappa$ as well as $\tau_{2} / \eta$ by $\tau_{1 \text { eff }} / \kappa$ and $\tau_{2 \text { eff }} / \eta$, respectively, in expressions (2.17a) for $r_{1}$ and $r_{3}$ and then inserting these values in Eq. (2.25) for $D(\omega, \phi)$ one is led to the new values for the critical Mach number. In model G II $\left[r_{1}=(2 / 5 \pi) 0.80, r_{2}=0\right.$, and $r_{3}=(2 /$ $5 \pi) 0.25]$ upon setting $D(\omega, \phi)=0$ one obtains the equation $(1-\phi)\left(2.4453 \phi^{2}-0.2281 \phi+0.0048\right)=0$. Its nontrivial roots are $\phi_{1}=0.0612$ and $\phi_{2}=0.0320$ and the corresponding critical Mach numbers, calculated via Eqs. (2.21) and (2.22), turn out to be $M_{c 1}=3.033$ and $M_{c 2}=4.259$, respectively.

Unfortunately, the normalization of $r_{2}$, the coefficient related to the entropy flow, appearing in model G I, requires more involved arguments based on fluctuation theory and we will not deal with it here but in a future work which is currently in progress.

\section{NONLINEAR EFFECTS: FOURTH-ORDER TERMS IN THE ENTROPY}

In the previous section only linear nonlocal effects were considered. Since a shock wave drives the medium far away from thermodynamic equilibrium with rather high values for the heat flow and the viscous pressure it seems quite natural to take into account nonlinear effects. In this section we examine a particular kind of nonlinear effect related to an expansion of the nonequilibrium entropy function up to fourth order in the heat flow and viscous pressure.

We now consider an expression for the nonequilibrium entropy analogous to the integrated form of (2.1),

$$
\rho s=\rho s_{\mathrm{eq}}-\frac{\tau_{1}}{2 \kappa T^{2}} q^{2}-\frac{\tau_{2}}{4 \eta T} \mathrm{P}^{v}: \mathrm{P}^{v},
$$

namely,

$$
\begin{aligned}
\rho s= & \rho s_{\mathrm{eq}}-\frac{\alpha}{2} q^{2}\left[1+\frac{\beta}{2 \alpha} q^{2}\right) \\
& -\frac{\alpha^{\prime}}{2} \mathrm{P}^{v}: \mathrm{P}^{v}\left[1+\frac{\beta^{\prime}}{2 \alpha^{\prime}} \mathrm{P}^{v}: \mathrm{P}^{v}\right) .
\end{aligned}
$$

This expression is the obvious generalization of (4.1a) up to fourth order in the flows. The coefficients $\alpha$ and $\alpha^{\prime}$ are those occurring in the second-order entropy and they are given by

$$
\alpha \equiv \frac{\tau_{1}}{\kappa T^{2}}, \quad \alpha^{\prime} \equiv \frac{\tau_{2}}{2 \eta T},
$$

with $\tau_{1}$ and $\tau_{2}$ the relaxation times of the linear theory. 
The coefficients $\beta$ and $\beta^{\prime}$ are new and related to the fourth-order contributions to the entropy.

By defining the effective nonlinear relaxation times $\tau_{1}^{*}$ and $\tau_{2}^{*}$ according to

$$
\begin{aligned}
& \tau_{1}^{*}=\tau_{1 \text { eff }}\left(1+\frac{\beta}{2 \alpha} q^{2}\right), \\
& \tau_{2}^{*}=\tau_{2 \text { eff }}\left(1+\frac{\beta^{\prime}}{2 \alpha^{\prime}} \mathrm{P}^{v}: \mathrm{P}^{v}\right),
\end{aligned}
$$

the relationship (4.1b) can be written as (4.1a) but with $\tau_{1}$ and $\tau_{2}$ replaced by $\tau_{1}^{*}$ and $\tau_{2}^{*}$, respectively. By $\tau_{1 \text { eff }}$ and $\tau_{2 \text { eff }}$ we denote the effective relaxation times, which take into account nonlocal effects only, evaluated in the previous section. $\tau_{1}^{*}$ and $\tau_{2}^{*}$ include both nonlocal corrections (coming from $\tau_{1 \text { eff }}$ and $\tau_{2 \text { eff }}$ ) and nonlinear corrections (coming from the terms in large parentheses).

The coefficient $\alpha, \beta$ as well as $\alpha^{\prime}$ and $\beta^{\prime}$ can be computed from fluctuation theory, in terms of the second- and fourth-order moments of the fluctuations around an equilibrium state [23]. They read

$$
\begin{aligned}
& \alpha=\frac{2}{5 p^{2} T}, \quad \beta=-\frac{862}{625} \frac{m}{p^{4} k_{B} T^{2}}, \\
& \alpha^{\prime}=\frac{1}{2 p T n m}, \quad \beta^{\prime}=-\frac{3}{8} \frac{k_{B}}{m p^{4}} .
\end{aligned}
$$

Hence the nonlinear-nonlocal effective relaxation times are

$$
\begin{aligned}
& \tau_{1}^{*}=\tau_{1 \text { eff }}\left(1-\frac{431}{250} \frac{m}{p^{2} k_{B} T} q^{2}\right), \\
& \tau_{2}^{*}=\tau_{2 \text { eff }}\left(1-\frac{3}{4} \frac{P_{x x}^{v 2}}{p^{2}}\right) .
\end{aligned}
$$

For a given Mach number, one may evaluate the heat flow $q$ and the viscous pressure $P_{x x}^{v}$ across the shock layer as

$$
q=-\kappa_{0} \frac{T_{-}-T_{+}}{L}, \quad P_{x x}^{v}=-\frac{4}{3} \eta_{0} \frac{v_{-}-v_{+}}{L},
$$

with $L$ the width of the shock layer and $\kappa_{0}$ and $\eta_{0}$ the values taken by $\kappa$ and $\eta$, respectively, at the center of the layer. We borrow these values from kinetic theory $\kappa_{0}=5 n k_{B}^{2} T \tau_{1} / 2 m, \eta_{0}=n k_{B} T \tau_{2}$. As a consequence one has

$$
\begin{aligned}
& \tau_{1}^{*}=\tau_{1 \text { eff }}\left(1-4.209 \frac{l_{-}^{2}}{L^{2}} \frac{\left(T^{*}-1\right)^{2}}{T^{*}+1}\right], \\
& \tau_{2}^{*}=\tau_{2 \text { eff }}\left(1-0.404 \frac{l_{-}^{2}}{L^{2}} M_{-}^{2} \frac{\left(v^{*}-1\right)^{2}}{v^{* 2}}\right),
\end{aligned}
$$

where $T^{*}$ and $v^{*}$ are short for $T_{+} / T_{-}$and $v_{-} / v_{+}$, respectively. For monoatomic ideal gases $\left(\gamma=\frac{5}{3}\right)$ one has

$$
T^{*}=\frac{\left(5 M_{-}^{2}-1\right)\left(M_{-}^{2}+3\right)}{16 M_{-}^{2}}, v^{*}=\frac{4 M_{-}^{2}}{M_{-}^{2}+3} .
$$

Thus, Eqs. (4.7) and (4.8) enable us to obtain the nonequilibrium corrections to the effective relaxation times as a function of the Mach number. Here we will take for $l_{-} / L$ the value $l_{-} / L \cong 1 / 4$ which is the observed value for that ratio for Mach numbers ranging from 2 to 5 (see Fig. 48 in Cercignani, Ref. [1]) and we will evaluate $r_{1}^{*}$ and $r_{3}^{*}$ in the equations. For model G II we get

$$
\begin{aligned}
& r_{1}^{*}=\frac{2}{5 \pi} 0.80\left[1-0.01644 \frac{A(M)}{B(M)}\right], \\
& A(M)=\left[\left(5 M_{-}^{2}-1\right)\left(M_{-}^{2}+3\right)-16 M_{-}^{2}\right]^{2}, \\
& B(M)=M_{-}^{2}\left[\left(5 M_{-}^{2}-1\right)\left(M_{-}^{2}+3\right)+16 M_{-}^{2}\right],
\end{aligned}
$$

and

$$
r_{3}^{*}=\frac{2}{5 \pi} 0.25\left[1-0.01519 \frac{\left(M_{-}^{2}-1\right)^{2}}{M_{-}^{2}}\right] .
$$

The ratio $2 / 5 \pi$ is the value of $r_{1}$ and $r_{3}$ according to model G II of Anile and Majorana [15], whereas 0.80 and 0.25 are the nonlocal corrections of the last section and the terms in large parentheses are the nonlinear corrections.

The method to follow is an iterative one. We compute the value of the nonlinear corrections [the terms in large parentheses in (4.9) and (4.10)] for $M_{-}=3$, the critical Mach number in the linear nonlocal theory, and obtain $r_{1}^{*}=0.0540$ and $r_{3}^{*}=0.0303$. Upon introducing these values back in (2.25) the nontrivial roots of $D(\omega, \phi)=0$ happens to be $\phi_{1}=0.0365$ and $\phi_{2}=0.0274$. According to these values the critical Mach number is 3.9797. Then using again the expressions (4.9) and (4.10) we compute the nonlocal-nonlinear corrections for $M_{-}=3.9797$ and obtain $r_{1}^{*}=0.03598$ and $r_{3}^{*}=0.2682$, respectively. The roots of $D(\omega, \phi)=0$ are now $\phi_{1}=0.0298$ and $\phi_{2}=0.0201$ and the critical Mach number increases up to 4.4202. In a subsequent step we get $r_{1}^{*}=0.009527, r_{3}^{*}=0.02503$, $\phi_{1}=0.0267$, and $\phi_{2}=0.00565$. The corresponding critical Mach number now is 4.67. At this point we stop the process for the next step leads to a negative value for $r_{1}^{*}$, which is clearly unphysical. Thus the nonlinear effects considered in this section have raised the critical Mach number from 3.03 up to 4.67 .

\section{CONCLUDING REMARKS}

The nonlinearities considered in last section are by no means the only ones conceivable. Other nonlinearities may arise. For instance, $\mathrm{Eu}$ has proposed in the framework of a modified moment method the following nonlinear corrections to the phenomenological transport coefficients [7]:

$$
\kappa\left(q, \mathrm{P}^{v}\right)=\kappa C(\chi), \quad \eta\left(q, \mathrm{P}^{v}\right)=\eta C(\chi),
$$

where $C(\chi)=\sqrt{\chi} / \sinh \sqrt{\chi}$ and $\chi$ denotes the dimensionless parameter

$$
\chi=\frac{\tau}{n k_{B}} \sigma_{\text {lin }}^{s} .
$$


Here $\tau$ stands for a relaxation time which we take as $\tau_{2}$ and $\sigma_{\text {lin }}^{s}$ denotes the classical entropy production

$$
\sigma_{\text {lin }}^{s}=-\mathrm{q} \cdot \nabla T^{-1}-\mathrm{P}^{v}: T^{-1} \mathrm{~V} .
$$

If one takes into account the correction proposed by $\mathrm{Eu}$ one should evaluate at each step $\sigma_{\text {lin }}^{s}$ as a function of the Mach number. In such a case expressions (4.9) and (4.10) should be replaced by

$$
\begin{aligned}
& r_{1}^{*}=\frac{2}{5 \pi} \frac{0.80}{C(\chi)}\left[1-0.01644 \frac{A(M)}{B(M)} C^{2}(\chi)\right], \\
& r_{3}^{*}=\frac{2}{5 \pi} \frac{0.25}{C(\chi)}\left[1-0.01519 \frac{\left(M_{-}^{2}-1\right)^{2}}{M_{-}^{2}} C^{2}(\chi)\right],
\end{aligned}
$$

with

$\chi=\frac{l_{-}^{2}}{L^{2}}\left[0.09155 \frac{A(M)}{B(M)}+0.3255 \frac{\left(M_{-}^{2}-1\right)^{2}}{M_{-}^{2}}\right]$.

These corrections shorten a bit the iterative method of the last section and only result in a slight increase of the critical Mach number.

In summary, we have shown how higher-order nonlocal and nonlinear effects may significantly raise the critical Mach number predicted by EIT. Linear nonlocal corrections raise its value from 2.01 up to 3.03. In turn, nonlinear corrections further increase the critical Mach number from 3.03 up to 4.67 .

Note that we stopped our iterative process when $r_{1}^{*}$ would become lower than zero for it would imply a negative relaxation time $\tau_{1}$ which is against causality. However, the critical Mach number could be much greater if for higher Mach numbers the ratio $l_{-} / L$ would decrease as $1 / M_{-}$, as it seems to be suggested by experimental results. If this would indeed happen, it would be possible to keep $r_{1}^{*}$ and $r_{3}^{*}$ positive [see Eqs. (4.9) and (4.10)] in spite of the raising of $M_{-}$.

We want to emphasize that expressions (4.9) and (4.10) are based on a fourth-order expansion of the nonequilibrium entropy function and higher-order expansions should be needed for a more accurate analysis. This would hopefully result in a further increase of the critical Mach number.

Before closing this paper it is worth noting that (in contrast to classical nonequilibrium theory) EIT sets a limit on its own range of applicability. A stability analysis based on the second-order fluctuations of the entropy out of equilibrium (see Micenmacher and Jou in Ref. [15]) shows that EIT may only be valid for values of the heat flow and the shear stress tensor lower than some critical values. This condition can be written in terms of the ratio between the upstream mean free path and the shock width as $l_{-} / L \geq 2$, a relationship fulfilled by shock waves at least up to Mach numbers as greater as four.

\section{ACKNOWLEDGMENTS}

One of us (D.P.) is greatly indebted to Professor A. M. Anile, Dr. A. Majorana, and Dr. O. Muscato for helpful discussions as well as the Seminario Matematico of the University of Catania, where part of this work was done, for warm hospitality and financial support. This work has been partially supported by the Spanish Ministry of Education under Grant No. PB89-0290.
*Electronic address: iftg1@ ccuab1.uab.es. Also at Institut d'Estudis Catalans, Barcelona, Spain.

[1] H. Grad, in Principles of Kinetic Theory of Gases, Handbuch der Physik XII, edited by S. Flugge (Springer, Berlin, 1958); C. Cercignani, The Boltzmann Equation and Its Applications (Springer, Berlin, 1988).

[2] A. Z. Askcasu and E. Daniels, Phys. Rev. A 2, 962 (1970); M. Grant and R. Desai, ibid. 25, 2727 (1982).

[3] D. Jou, J. Casas-Vázquez, and G. Lebon, Rep. Prog. Phys. 51, 1104 (1988); Recent Developments in Nonequilibrium Thermodynamics, edited by J. Casas-Vázquez and G. Lebon (Springer, Berlin, 1984); G. Lebon, D. Jou, and J. Casas-Vázquez, J. Phys. A 13, 275 (1980).

[4] I. Muller, Z. Phys. 198, 329 (1967); I. S. Liu and I. Muller, Arch. Ration. Mech. Anal. 83, 320 (1983).

[5] L. S. Garcia Colin, Rev. Mex. Fis. 34, 344 (1988); L. S. Garcia Colin, M. López de Haro, F. Rodriguez, J. CasasVázquez, and D. Jou, J. Stat. Phys. 37, 465 (1984).

[6] R. E. Nettleton, Phys. Fluids 2, 256 (1959); 3, 216 (1960); J. Phys. A 21, 3939 (1988).

[7] B. C. Eu, J. Chem. Phys. 71, 4832 (1979); 73, 2958 (1980).

[8] Advances in Thermodynamics, edited by S. Salomon and S. Sieniutycz (Francis and Taylor, New York, 1991), Vol. VI.

[9] Z. Banach, J. Math. Phys. 30, 1804 (1989); 30, 1816 (1989).

[10] M. Grmela and G. Lebon, J. Phys. A 23, 3341 (1990); M. Grmela and D. Jou, J. Phys. A 24, 741 (1991).

[11] D. D. Joseph and L. Preziosi, Rev. Mod. Phys. 61, 41 (1989); 62, 375 (1990).
[12] D. Jou, J. E. Liebot, and J. Casas-Vázquez, Phys. Rev. A 25, 508 (1982); D. Jou and T. Careta, J. Phys. A 15, 3195 (1982).

[13] M. Criado-Sancho, D. Jou, and J. Casas-Vázquez, Macromolecules 24, 2834 (1991).

[14] W. Israel, Ann. Phys. 100, 310 (1976); W. A. Hiscock and L. Lindblom, Phys. Rev. D 31, 725 (1985); D. Pavón, D. Jou, and J. Casas-Vázquez, Ann. Inst. Henri Poincaré A 36, 79 (1982); 42, 31 (1985).

[15] A. M. Anile and A. Majorana, Meccanica 16, 149 (1981); V. Micenmacher and D. Jou, Phys. Lett. A 141, 165 (1989); G. Lebon and A. Cloot, Wave Motion 16, 23 (1981).

[16] M. Linzer and D. F. Hornig, Phys. Fluids 6, 166 (1963).

[17] G. A. Bird, J. Fluid Mech. 30, 479 (1967).

[18] W. Israel, in Relativistic Fluid Dynamics, edited by A. M. Anile and $Y$. Choquet-Bruhat, Lecture Notes in Mathematics Vol. 1385 (Springer, Berlin, 1988).

[19] C. Pérez-Garcia and D. Jou, J. Phys. A 19, 2881 (1986); Phys. Lett. 107A, 390 (1985).

[20] M. Carrassi and A. Morro, Nuovo Cimento B 9, 321 (1972); 13, 281 (1973).

[21] S. Hess, Z. Naturforsch. Teil A 32, 678 (1977).

[22] P. Gianozzi, C. Grosso, S. Moroni, and G. PastoreParravicini, Appl. Num. Math 4, 273 (1988).

[23] M. Ferrer, Doctoral thesis, Autonomous University of Barcelona, 1991; M. Ferrer and D. Jou (unpublished). 